\title{
Outcome of tension free repair of incisional hernia
}

\author{
Mohamed H. El-Ghor, MD; M.A.Marzouk, MD; Ehab H. Abd El-Wahab, MD; \\ Mohamed Rady, MD
}

Departmrnt of General Surgery, Ain Shams University, Cairo, Egypt.

Background: The surgical treatment of incisional hernia has changed rapidly during the last decade with the increasing use of mesh technique and the introduction of laparoscopy. However, many questions concerning mesh type, mesh positioning, fixation method and operation type still remain unanswered.

Methods: The study includes 100 cases of anterior abdominal wall hernias, including incisional hernia (cicatricial or paralytic types). The idea of the tension free repair of anterior abdominal wall hernia depends on repair of the defect by double layer onlay polypropylene mesh the deeper mesh is fixed to the free edge of the defect by polypropylene (No. 1). The mean operative time was 2 hours (ranging between 1.5 - 4 hours). Follow up of the patients was performed every 3 month with maximum follow up of two years and a minimum of six months.

Results: The early postoperative course was uneventful in 96 patients (96\%). Four patients presented with wound infection, two patients presented with superficial infection, the other two patients presented with deep infection and exposed mesh. The follow up revealed 8 cases of recurrent hernia (8\%), two of these cases were associated with obesity, and one case was associated with bronchial asthma.

Conclusion: In our study we modified the onlay technique for incisional hernia repair by using double layer onlay polypropylene mesh the deeper mesh is fixed to the free edge of the external oblique muscle, this modification insured a tension free repair of the hernia, the technique is universal and can be applied in both scar and paralytic insicional hernia.

Key words: Incisional hernia, tension free repair, onlay repair.

\section{Introduction:}

The field of hernia surgery has changed markedly within the past decade. Today, every patient and every surgeon has the choice between various techniques and devices to repair incisional hernias. Lots of publications confirm that most of them can be applied with success. The overall low recurrence rates published make it difficult to decide, which one is the best. ${ }^{1}$

Thousands of laparotomy incisions are performed each year, the failure rate for closure of these abdominal wounds is between $10-15 \%$, thus creating the overwhelming problem of incisional hernia. ${ }^{2}$

Incisional hernia is defined as "Any abdominal wall gap with or without bulge in the area of a postoperative scar perceptible or palpable by clinical examination or imaging". 3

The risk of acquiring incisional hernia is heightened by several risk factors including obesity, diabetes, emergency surgery, postoperative wound dehiscence, smoking and postoperative wound infection. ${ }^{4}$

Inscisional hernia inflicts at least 400,000 to 500,000 patients in the United States alone per year. The burden of incisional hernias varies widely but primarily comprises pain at the site of the hernia, limitations of activity, intestinal obstruction, skin ulceration, and even death due to compromise of the intestinal blood supply have been reported. 5

When morbidity is added to the huge numbers and the tremendous costs associated with incisional hernia repair, it becomes clear 
that the efficacy of incisional hernia repair is of major importance. ${ }^{6}$

Prevention of incisional hernia presents a difficult challenge owing to the multifactorial nature of its incidence; weight reduction and treatment of predisposing factors and preexisting illnesses is advised before abdominal surgery. ${ }^{7}$

Several classifications of hernia were suggested, based on localization, size, recurrence, reducibility and presenting symptoms. ${ }^{3}$

The indications of incisional hernia repair range from cosmoses, pain and discomfort to irreducibility, narrow neck and history of recurrent subacute intestinal obstruction, whereas strangulation and obstruction pose an absolute indication for surgery. ${ }^{8}$

At present, different operative techniques of hernioplasty are used, such as simple closure, Mayoduplication, prosthetic-implantation in the onlay and sublay techniques, autodermal plasty as well as laparoscopic procedures. ${ }^{9}$

The introduction of prosthetic mesh repair has revolutionized the repair of incisional hernia and rendered obsolete most of the older methods of repair, ${ }^{10}$ the search for the ideal prostheses is continuous and oriented towards the use of low weight, large pore polypropylene prostheses which enhances proper tissue integration. ${ }^{11}$

Unfortunately, results of incisional hernia repair are disappointing, recurrence rates reach up to $49 \%$ in open suture repair, and up to $10 \%$ in open and laparoscopic mesh repair. ${ }^{12}$

Continuous efforts are being held to reach the optimum management of incisional hernia, so far the most important prognostic factor influencing the outcome is the surgeon's experience. ${ }^{13}$

The treatment of incisional hernia is a current problem in modern surgery. Many important aspects of incisional hernia surgery are yet to be answered, especially the choice of surgical technique and its adaptation to the individual patient. ${ }^{3}$

\section{Patients and methods:}

The study includes 100 cases of anterior abdominal wall hernias, including incisional hernia (cicatricial or paralytic types). The recurrent cases are included in the study, the patients were operated on between 2010 and 2012.

Selection Criteria: Patients with incisional hernias (cicatricial or paralytic type) and recurrent cases are included.

Operative technique: The idea of the tension free repair of anterior abdominal wall hernia depends on the followings: (1) Proper dissection of the sac. (2) Proper identification of the defect Figure (1,2). (3) Reduction of the content. (4) Removal of the excess sac and reclosure to have an intact peritoneum under the mesh. (5) Repair of the defect by double layer onlay polypropylene mesh the deeper one is fixed to the free edge of the defect by continuous polypropylene (No. 1) sutures, the superficial one is inserted and fixed $3 \mathrm{~cm}$ from the edge of the defect superificial to the deeper mesh.

The postoperative analysis included: The patients were followed in the early postoperative by the amount of the discharge in the suction drain and the condition of the skin flaps.

The drain was removed in the $7^{\text {th }}$ postoperative day and the stitches after 3 weeks and the patient was re examined every 3 months as regards the tension of the repair and any sinuses along the suture line. The patient was encouraged to start abdominal exercise gradually after 3 month.

\section{Results:}

Patient demographics: Hernia reconstruction was performed on 100 patients, 69 females (69\%) and 31 males (31\%) with incisional hernias (cicatricial or paralytic type) and recurrent cases, the mean age of the patients was 38 (range 22-62 years).

The mean body mass index was $28.2 \mathrm{~kg} /$ $\mathrm{m}^{2}$, the mean length of the defect was $12 \mathrm{~cm}$, the mean width of the defect was $7 \mathrm{~cm}$.

Associated co-morbidities included diabetes, hypertension, cirrhosis, bronchial asthma, ischemic heart disease and atherosclerosis as well as obesity Table (1).

Tension free repair of the abdominal 


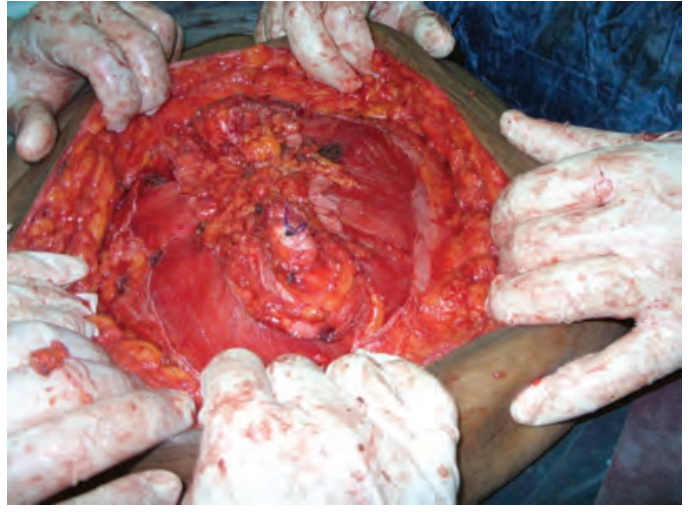

Figure (1): Dissection of the sac and identification of the defect.

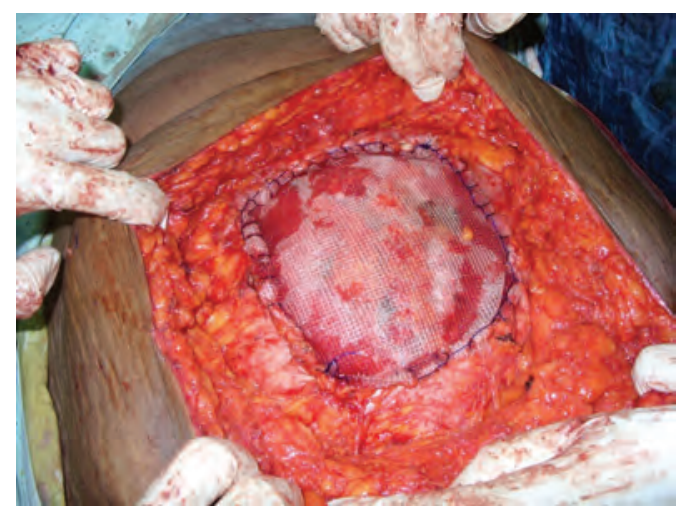

Figure (3): The deeper mesh is sutured to the edge of the defect in the external oblique muscle by continous polypropylene sutures.

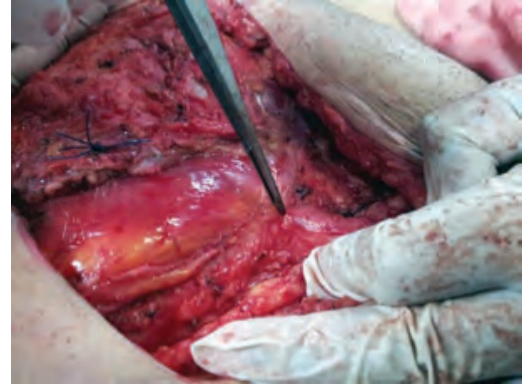

Figure (2): Proper identification of the edge of the defect is mandatory as the deeper mesh will be sutured to the edge of the defect.

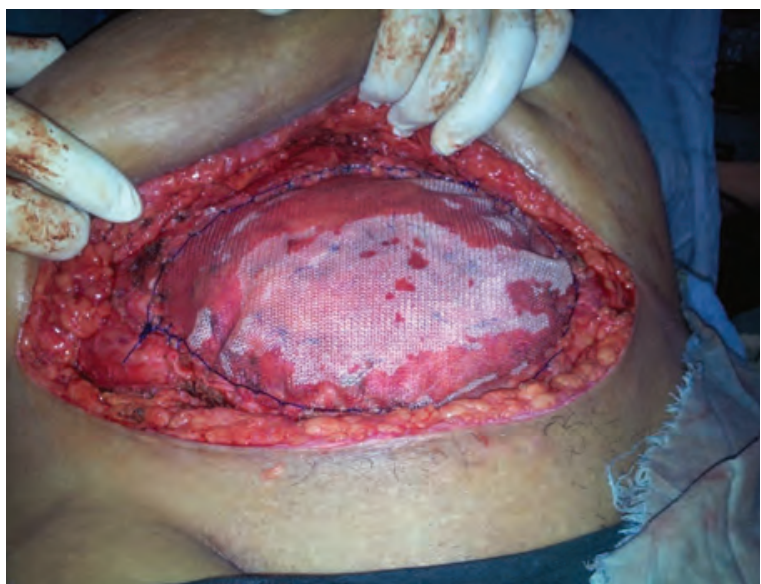

Figure (4): Relatively larger defect repaired by suturing the deeper mesh to the well dissected edge of the external oblique muscle.

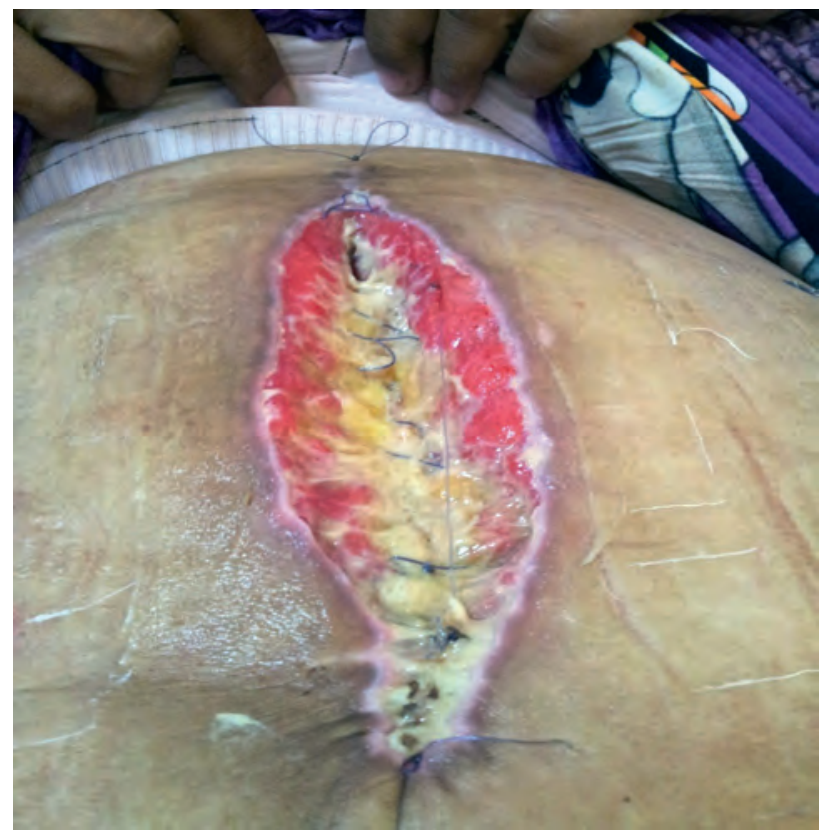

Figure (5): Superficial wound infection treated by repeated dressing. 
Table (1): Demographic data, Clinical characteristics and associated comorbidities.

\begin{tabular}{|l|l|}
\hline \multicolumn{2}{|l|}{ Age “ years” } \\
\hline Range & $22-62$ \\
\hline Mean & 38 \\
\hline \multicolumn{2}{|l|}{ Sex no. of patients (\%) } \\
\hline Female (\%) & $69(69 \%)$ \\
\hline Male \% & $31(31 \%)$ \\
\hline BMI & \\
\hline Range & $24-33$ \\
\hline Mean & 28.2 \\
\hline Obese >30 & 19 \\
\hline D.M. & 24 \\
\hline Hypertension & 20 \\
\hline Cirrhosis & 3 \\
\hline Bronchial asthma & 3 \\
\hline Ischemic heart & 4 \\
\hline Atherosclerosis & 5 \\
\hline
\end{tabular}

defect was performed on the patients in the following order: proper dissection of the sac and reduction of the content, reclosure of the defect with preservation of the intact peritoneum, followed by repairing the defect by double layer onlay polypropylene mesh fixed to the free edge of the defect by continous polypropylene sutures.

The site of the defect was either midline in 65 patients, following Fannestiel's incision in 33 patients, following Kocher's incision in a single patient and following Mcburney's incision in a single patient Table (2).

Reconstruction was performed under clean conditions in 98 patients and cleancontaminated in two patients.

The mean operative time was 2 hours (ranging between $1.5-4$ hours).

Postoperative course: The early postoperative course was uneventful in 96 patients (96\%). Four patients presented with wound infection, two patients presented with superficial infection Figure (5) which was properly managed by regular wound dressing for three weeks, the other two patients
Table (2): Type, size and length of the defect.

\begin{tabular}{|l|l|}
\hline Type of hernia & \\
\hline Cicatricial & 54 \\
\hline Paralytic & 34 \\
\hline Recurrent & 12 \\
\hline $\begin{array}{l}\text { Length of the } \\
\text { defect }\end{array}$ & \\
\hline Range & $8-17$ \\
\hline Mean & 12 \\
\hline $\begin{array}{l}\text { Width of the } \\
\text { defect }\end{array}$ & \\
\hline Range & $5-11$ \\
\hline Mean & 7 \\
\hline Site of the defect & \\
\hline Midline & 65 \\
\hline Fannestiel & 33 \\
\hline Kocher & 1 \\
\hline Macburney & 1 \\
\hline
\end{tabular}

presented with deep infection and exposed mesh, they were adequately managed by secondary suturing after two weeks, the sutures were removed after ten days.

Follow up of the patients was performed every 3 month with maximum follow up of two years and a minimum of six months, the follow up revealed 8 cases of recurrent hernia (8\%), two of these cases were associated with obesity, and one case was associated with bronchial asthma.

\section{Discussion:}

The surgical treatment of incisional hernia has changed rapidly during the last decade with the increasing use of mesh technique and the introduction of laparoscopy. However, many questions concerning mesh type, mesh positioning, fixation method and operation type still remain unanswered. Patients with incisional hernia are a heterogeneous population with patient-specific co-morbidity and innate differences (e.g. collagen formation quality). This makes the choice of technique most suitable for each patient even more 
difficult. ${ }^{14}$

The onlay technique is one of the established techniques of surgical treatment of incisional hernia, it was first described as prefascial prosthetic implantation by Chevrel and Rath. ${ }^{15}$

In our study we modified the onlay technique for insicional hernia repair by using double onlay polyprolene mesh where the deeper mesh is fixed to the free edge of the external oblique muscle. The superficial mesh was inserted and fixed $3 \mathrm{~cm}$ from the edge of the defect superificial to the deeper mesh.

This modification insured a tension free repair of the hernia, the technique is universal and can be applied in both scar and paralytic insicional hernia.

Another advantage to this technique is the possibility of its application in different sites, in our study we applied the technique successfully in midline hernia as well incisional hernia following Fannestiel's, Kocher's and Mcburney's incisions.

In concordance with the component separation technique, the onlay technique with release of the external oblique muscle is successful in large midline and recurrent hernias, Many surgeons already recommend the additional application of synthetic mesh in an onlay position to supplement the attenuated layers of the anterior abdominal wall, ${ }^{2}$ however the component separation technique requires an undamaged rectus muscle, it also carries a risk of injuring the blood supply and the nerves that run between the internal oblique and transverse muscle. ${ }^{16}$

By applying the mesh in an onlay fashion, we avoided the drawbacks in the inlay technique, represented by the induction of extensive adhesions of viscera if placed in a position where they become adjacent to bowel, erosion of the mesh may then occur into the intestines, ${ }^{2}$ the onlay technique with muscle release can possibly substitute the inlay technique in the management of huge incisional hernia.

The complex sublay technique is limited to the management of midline hernias and, in the lower one-third of this region, the mesh is only protected from bowel by weak peritoneum carrying the risk of inducing adhesions. ${ }^{17}$

As regard postoperative complications in our study four cases of wound infection were reported (4\%) no postoperative mesh removal was performed compared to a rate of wound healing complications after simple onlay operation ranging between $4 \%$ and $26 \%$ and an estimated rate of prosthesis removals between $0 \%$ and $2.5 \% .{ }^{16}$

Regarding the literature, the recurrence rates and the percentage of wound healing complications between the onlay and sublay techniques are comparable, the sublay technique is more complicated and requires an experienced and high skilled surgeon. ${ }^{3}$

The recurrence rate in our present study was $8 \%$, in a comparative study De Vries Reilingh et al, $2004^{16}$ compared the inlay technique with onlay and sublay in 53 patients with large midline incisional hernia. In this series, the recurrence rate was significantly higher in the inlay technique (44\%) compared to $28.3 \%$ in the onlay technique and $12 \%$ in sublay technique.

The recurrence rates of the onlay technique indicated in the literature vary between $2.5 \%$ and $13.3 \%$. Some recurrences after onlay implantation result from a peripheral mesh dislocation, or an insufficient size of the mesh. So-called "subprosthetic hernia" is possible in cases of the combination of the fascia rupture and laxity of the anterior abdominal wall. ${ }^{3}$

The recurrence rate in the component separation technique was rather inconsistent, in a relatively large series of 43 patients, De Vries Reilingh and colleagues, $2003^{16}$ were unable to reproduce the good results of Ramirez et al., $1990^{18}$ and recorded recurrent hernia in 32\% of patients at 15-month followup. Reherniation was assumed to be the result of insufficient release of the external oblique muscle at its insertion on the thoracic wall.

DiBello and Moore, $1996^{19}$ used a modified component separation technique in 35 patients, in 15 patients midline closure was supported by an onlay prosthesis of expanded polytetrafluoroethylene or a Vicryl mesh. Reherniation was found in 9\% after a mean followup of 22 months.

The simple reconstruction has an 
unacceptably high recurrence rates ranging between $25 \%$ and $55 \%$. Because of these high rates after simple reconstruction and the development of new tissue-compatible, prosthetic materials, many surgeons share the opinion that an additional strengthening of the frontal abdominal wall by implantation of allo- and autoplastic material should be obligatory. ${ }^{3}$

\section{Conclusion:}

In our study, we modified the onlay technique for incisional hernia repair by using double layer onlay polypropylene mesh the deeper mesh is fixed to the free edge of the external oblique muscle, the superficial one is inserted and fixed $3 \mathrm{~cm}$ from the edge of the defect superificial to the deeper mesh, this modification provided a tension free repair of the defect.

The advantages of this technique is its versatility as it can be applied to different sites of incisional hernia, also it works well with large midline hernias, the study included cicatricial and paralytic incisional hernia as well as recurrent cases.

The recurrence rate was acceptably low (8\%) with minimal postoperative complications, we recorded (0) mortality and no cases of mesh removal.

We conclude that onlay repair of incisional hernia with release of the external oblique provides a good and versatile alternative for management of large midline hernias as well as other sites.

\section{Reference:}

1- Conze J, Binnebösel $M$, Junge $K$, et al: Incisional hernia: How do I do it? Standard surgical approach. Chirurg 2010; 8(3): 192-200.

2- Kingsnorth A: The management of incisional hernia. Ann R Coll Surg Engl 2006; 88(3): 252-260.

3- Korenkov M, Paul A, Sauerland S et al: Classification and surgical treatment of incisional hernia. Results of an experts' meeting. Langenbecks Arch Surg 2001; 386(1): 65-73.

4- Wong SY, Kingsnorth AN: Prevention and surgical management of incisional hernias. Int J Surg Invest 2001, 3: 407-414.
5- Flum DR, Horvath K, Koepsell T: Have outcomes of incisional hernia repair improved with time? A population-based analysis. Ann Surg 2003; 237(1): 129-135.

6- DeMaria EJ, Moss JM, Sugerman HJ: Laparoscopic intraperitoneal polytetrafluoroethylene (PTFE) prosthetic patch repair of ventral hernia. Prospective comparison to open prefascial polypropylene mesh repair. Surg Endosc 2000; 14(4): 326-329.

7- Cassar K, Munro A: Surgical treatment of incisional hernia. Br J Surg 2002; 89(5): 534-545.

8- O’Dwyer PJ, Courtney CA: Factors involved in abdominal wall closure and subsequent incisional hernia. Surgeon. 2003. 1(1): 17-22.

9- Chevrel JP , Rath AM: Classification of incisional hernias of the abdominal wall. Hernia 2000; 4: 7-11.

10- Adotey JM: Incisional hernia: A review. Niger J Med. 2006; 15(1): 34-43.

11- Schumpelick V, Klosterhalfen B, Müller M, et al: Minimized polypropylene mesh for preperitoneal net plasty (PNP) of incisional hernias. Chirurg 1999; 70(4): 422-430.

12- Chan G, Chan CK: A review of incisional hernia repairs: Preoperative weight loss and selective use of the mesh repair. Hernia 2005; 9: 37-41.

13- Langer C, Schaper A, Liersch T, et al: Prognosis factors in incisional hernia surgery: 25 years' of experience. Hernia 2005, 9:16-21.

14- Andersen LP, Klein M, Gögenur I, et al: Long-term recurrence and complication rates after incisional hernia repair with the open onlay technique. BMC Surg 2009; 28: 9-6.

15- San Pio JR, Damsgaard TE, Momsen D, et al: Repair of giant incisional hernias with polypropylene mesh: a retrospective study. Scand $J$ Plast Reconstr Surg Hand Surg 2003; 37: 102-106.

16- De Vries Reilingh TS, van Goor H, Rosman C, et al: "Components separation technique" for the repair of large abdominal wall hernias. J Am Coll Surg 2003; 196(1): 32-37.

17- Fernandez RL, Garcia JS, Ortego PD, et al: Tissucol application in dermolipectomy and incisional hernia repair. Int Surg 2001; 86: 240-245.

18- Ramirez OM, Ruas E, Dellon AL: 'Components separation' method for closure of abdominal-wall defects: An anatomic and clinical study. Plast Reconstr Surg 1990; 86: 
519-526.

19- DiBello JN, Moore JH: Sliding myofascial flap of the rectus abdominis muscle for the closure of recurrent ventral hernias. Plast Reconstr Surg 1996; 98: 464-469. 